\title{
Medical Assistance in Dying (MAID) and the Neurosurgeon: Position Statement of the Canadian Neurosurgical Society (CNSS)
}

\author{
Sean Barry, Chris Ekong, Brian W. Wheelock, Richard Moulton, Peter Gorman, \\ Kesh Reddy, Ian Fleetwood
}

Keywords: Neurosurgery, Ethics, Legal Issues, Doctor-Patient Relationship

doi:10.1017/cjn.2017.51

Can J Neurol Sci. 2017; 44: 744-746

\section{Who We Are}

The Canadian Neurosurgical Society (CNSS) was established in 1965 to represent neurosurgeons nationally. The CNSS has approximately 300 members representing neurosurgeons and neurosurgery residents in Canada. The mission of the CNSS is to enhance the care of patients with diseases of the nervous system through education, advocacy, and improved methods of diagnosis, treatment, and rehabilitation. The CNSS is a member of the larger Canadian Neurological Sciences Federation, which represents the interests of patients encompassing the entire spectrum of neurological disease across the country.

This position statement was generated by the CNSS subcommittee on Medical Assistance in Dying (MAID), consisting of the authors of this manuscript. In addition, the contents of the position statement were informed by a CNSS members' survey on MAID (results to be published separately) as well as a CNSS members' forum on MAID that took place at the annual CNSS meeting in Quebec City on June 21, 2016.

\section{Background: Supreme Court Judgment on MAID AND BILL C-14}

On February 6, 2015, the Supreme Court of Canada (SCC) rendered a decision striking down the Criminal Code absolute prohibition on providing assisted dying. The SCC's conclusion that "physician assisted death for a competent adult person who (1) clearly consents to the termination of life and (2) has a grievous and irremediable medical condition (including an illness, disease or disability) that causes enduring suffering that is intolerable to the individual in the circumstances of his or her condition" shall no longer be prohibited by law. The court suspended this decision for 12 months to allow for legislators and regulators to enact appropriate legislation. ${ }^{1}$ A second, 6-month extension to August 6, 2016, was requested by the Government of Canada to the SCC on December 3, 2015.

Bill C-14, legislation on MAID, received royal assent on June $17,2016 .^{2}$ This bill represents an amendment to the criminal code prohibiting MAID and, among other things, defines the eligibility criteria for MAID in Canada. Eligibility requires that all of the below criteria must be met:

A. patients are eligible for health services funded by a government in Canada;
B. they are at least 18 years of age and capable of making decisions with respect to their health;

C. they have a grievous and irremediable medical condition;

D. they have made a voluntary request for medical assistance in dying that, in particular, was not made as a result of external pressure; and

E. they give informed consent to receive medical assistance in dying after having been informed of the means that are available to relieve their suffering, including palliative care.

Bill C-14 goes on to provide that a person has a "grievous and irremediable medical condition" if all of the following are met:

A. they have a serious and incurable illness, disease, or disability;

B. they are in an advanced state of irreversible decline in capability;

C. that illness, disease, or disability or that state of decline causes them enduring physical or psychological suffering that is intolerable to them and that cannot be relieved under conditions that they consider acceptable; and

D. their natural death has become reasonably foreseeable, taking into account all of their medical circumstances, without a prognosis necessarily having been made as to the specific length of time that they have remaining.

This definition of a "grievous and irremediable medical condition" is a clear departure from the SCC's less restrictive definition in Carter $^{1}$ and essentially requires that the medical condition be terminal, and that natural death be either imminent or "reasonably foreseeable." Bill C-14 provides no guidance on how to determine what constitutes "reasonably foreseeable."

From the Division of Neurosurgery (SB), Dalhousie University, Halifax, Nova Scotia, Canada; Division of Neurosurgery (CE), Regina General Hospital, Regina,

Saskatchewan, Canada; Horizon Health Network, Neurosurgery (BWW), Saint John Regional Hospital, Saint John, New Brunswick, Canada; Division of Neurosurgery (RM) University of Ottawa, Ottawa, Ontario, Canada; Division of Neurosurgery (PG), The

Moncton Hospital, Moncton, New Brunswick, Canada; Department of Surgery (KR),

McMaster University, Hamilton, Ontario, Canada; Division of Neurosurgery (IF),

Island Health, Victoria, British Columbia, Canada.

Received November 16, 2016. Final Revisions Submitted March 1, 2017, Date of Acceptance March 25, 2017.

Correspondence to: Sean Barry, Division of Neurosurgery, Dalhousie University, Halifax, NS, CAN B3H 4R2. Email: spbarry@dal.ca 


\section{Definitions: Physician-Assisted Death, Euthanasia, And Palliative Care}

Physician-assisted death is the act of intentionally killing oneself with the assistance of a physician who provides the knowledge, means, or both. For the purposes of this discussion, physicianassisted death is analogous to MAID, which is the terminology the Government of Canada has elected to use. This differs from euthanasia, which is the deliberate action undertaken by any individual (including a physician) with the intention of ending the life of another person to relieve that person's suffering. Euthanasia may be further divided into voluntary euthanasia (with the consent of the patient) and nonvoluntary euthanasia (without the consent of the patient).

The World Health Organization has defined palliative care as a medical approach that improves the quality of life of patients and their families facing the problems associated with life-threatening illness, through the prevention and relief of suffering by means of early identification and assessment and treatment of pain and other problems; physical, psychosocial, and spiritual. Palliative care intends neither to hasten nor postpone death. It does not intend to cure disease but rather affirms life and regards dying as a normal process. ${ }^{3}$

\section{Position Statement}

Neurosurgical patients are a heterogeneous population encompassing all age groups. End-of-life decisions are commonly encountered by neurosurgeons because of the nature of their practice. Furthermore, many patients are not terminally ill but suffer from severe, medically intractable pain that may fit the criteria of "grievous and irremediable suffering." Although in certain situations death may be seen as a favorable or desirable outcome, it has never before been accepted as the goal of treatment. The recent SCC decision and subsequent change to the federal law through Bill C-14 heralds a change that will radically alter this most basic tenet that has guided physicians and surgeons for as long as the medical profession has existed.

Several high-profile neurosurgical patients have championed the cause of MAID in North America. Kay Carter, whose family filed the landmark lawsuit with the SCC mentioned previously, reportedly suffered from "spinal stenosis." A review of her symptoms reported in the lay-press, suggests that she likely suffered from degenerative cervical myelopathy. Brittany Maynard was diagnosed with an intracranial glioblastoma multiforma at the age of 29. She travelled from her home state of California to have MAID in Oregon in 2014. Her case received a tremendous amount of attention and was instrumental in changing the physicianassisted death laws in California in 2016.

Clinical situations in which MAID is requested are particularly challenging given the emotional, legal, and ethical issues involved. These matters are of particular importance to neurosurgeons, who care for a disproportionately high percentage of patients in severe, chronic pain or who suffer permanent disability. Many patients may see the choice of a medically assisted death as a compelling option.

The CNSS echoes the sentiments of the Canadian Society of Palliative Care Physicians that many requests for MAID are indications of suffering that could be ameliorated by palliative care. To that effect, the CNSS position is that all patients should be treated with respect and compassion. Furthermore, we feel that appropriate and effective palliative care should be the first line of treatment sought.
The CNSS supports the respect for patient autonomy; this means that competent patients have the right to accept or refuse any medical or surgical treatment offered or recommended to them. We support the Canadian Medical Association (CMA) statement that "All the requirements for informed consent must clearly be met, including the requirement that the patient be capable of making that decision, with particular attention to the context of potential vulnerabilities and sensitivities in end of life circumstances." We are particularly concerned with the impact this ruling may have on vulnerable populations. Effective safeguards and oversight is fundamental to the protection of these patients. The issue of oversight is particularly germane given recent reported findings from Belgium and the Netherlands regarding MAID, which were published after the SCC decision, suggesting safeguards enacted in these countries may be failing. ${ }^{4,5}$

The CNSS encourages a formalized process for mandated individualized patient education before embarking on the path to MAID. This would ensure that patients have adequate information from a medical expert pertaining to their particular illness regarding natural history; medical, surgical, interventional, endovascular, or other treatment modalities; treatment success rates; long-term efficacy; and procedural complications.

The CNSS affirms the belief that although the recent SCC decision on MAID may make it legally permissible to perform such an act, it does not make it ethically or morally acceptable 'ipso facto'.

The CNSS endorses the CMA's federal panel on MAID in its appeal for clarity on all legislation relating to MAID. There should be no "grey areas" on the requirements for qualification of MAID. The concepts of "grievous and irremediable suffering" and a "reasonably foreseeable death" laid out in Bill C-14 remain excessively vague and imprecise.

\section{Conscientious OBJection}

When a physician refuses to provide, or participate in, a legally recognized medical treatment or procedure because it conflicts with his or her own personal beliefs and values, this constitutes a "conscientious objection." MAID remains an ethically contentious issue. Experienced, compassionate physicians with deep moral convictions exist on both sides of the debate regarding the legalization of MAID. In an effort to respect this diversity of conviction, the CNSS supports the right to "conscientious objection" by physicians in clinical situations in which MAID conflicts with his or her own personal beliefs.

The CNSS opposes the view that the patient's "right to die" implies a corresponding responsibility on the part of any physician to actively participate/provide assistance in this process. A physician who makes a conscientious objection to participating in MAID should make every effort to minimize the disruption in the delivery of care to the patient. The physician should personally inform the requesting patient of their objection without imposing his or her own values on the patient. We support the following CMA statement: "In order to reconcile physicians' conscientious objection with a patient's request for access to assisted dying, physicians are expected to provide the patient with complete information on all options available to them, including assisted dying, and advise the patient on how they can access any separate central information, counseling, and referral service."

The CNSS supports the creation of the previously mentioned parallel and independent "separate central information, 
counseling and referral service" that can be accessed by patients, families, and allied health professionals. This type of accessible system, in contrast to a "duty to refer" process, would alleviate much of the moral dilemma faced by some physicians who may equate referral with participation. Furthermore, we support a clear separation between the neurosurgeon and the neurosurgical team and those physicians or allied health members involved in MAID. A clear delineation between the neurosurgical team and the MAID service will mitigate any potential for treatment bias, whether it be real or perceived. An analogy here may be made with current organ donation and tissue procurement services. To maintain a level of impartiality, a separate service is involved in this sensitive area with little formal overlap between the treatment team (e.g. the intensive care unit) and the organ donation service. There may be clinical situations in which the attending neurosurgeon volunteers to be actively involved in MAID, and the CNSS supports the right of its members to do so. Additionally, we feel that each individual institution should have an independent oversight committee to review each case of MAID before the process is carried out. This final step would ensure that all procedures have been followed and safeguards enacted before the ending of a life.

The CNSS strongly supports a clearly defined role for medical students and residents in the previously mentioned MAID service or team that allows these trainees to participate voluntarily. Trainees should not be allowed to enter into an agreement with a patient for MAID.

\section{Conclusion}

The CNSS welcomes the ongoing attention being given by Canadians to the suffering of patients. Above all, we urge further clarity in legislation regarding MAID and strict oversight in its implementation to reduce potential harm. We also support the creation of an independent, third-party referral service that would serve to respect the conscience of those health care providers who do not wish to actively participate in MAID.

\section{Disclosures}

The authors have nothing to disclose.

\section{REFERENCES}

1. Carter v. Canada (Attorney General), 2015 SCC 5, [2015] 1 S.C.R. 331.

2. Bill C-14, An Act to amend the Criminal Code and to make related amendments to other Acts (medical assistance in dying), 1st Session, 42nd Parliament. Available at http://laws-lois.justice. gc.ca/PDF/2016_3.pdf.

3. World Health Organization. WHO Definition of Palliative Care. Available at http://www.who.int/cancer/palliative/definition/en/.

4. Snijdewind MC, Willems DL, Deliens L, Onwuteaka-Philipsen BD, Chambaere K. A study of the first year of the end-of-life clinic for physician-assisted dying in the Netherlands. JAMA Intern Med. 2015;175:1633-40.

5. Dierickx S, Deliens L, Cohen J, Chambaere K. Comparison of the expression and granting of requests for euthanasia in Belgium in 2007 vs 2013. JAMA Intern Med. 2015;175:1703-6. 